NIST Special Publication 1200-17

\title{
Strategies for scanning electron microscopy sample preparation and characterization of multiwall carbon nanotube polymer composites
}

Version 1.0

András E. Vladár

This publication is available free of charge from:

http://dx.doi.org/10.6028/NIST.SP.1200-17

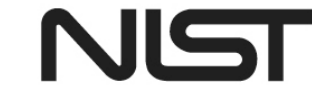

National Institute of Standards and Technology U.S. Department of Commerce 
NIST Special Publication 1200-17

\title{
Strategies for scanning electron microscopy sample preparation and characterization of multiwall carbon nanotube polymer composites
}

\author{
Version 1.0
}

\author{
András E. Vladár \\ Engineering Physics Division \\ Physical Measurement Laboratory
}

This publication is available free of charge from:

http://dx.doi.org/10.6028/NIST.SP.1200-17

July 2015

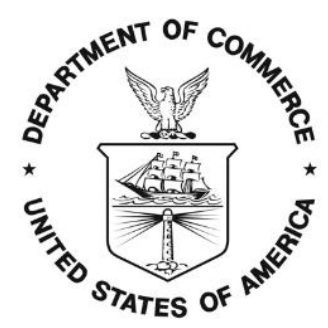

U.S. Department of Commerce

Penny Pritzker, Secretary

National Institute of Standards and Technology Willie May, Acting Under Secretary of Commerce for Standards and Technology and Acting Director 

Certain commercial entities, equipment or materials may be identified in this document in order to describe an experimental procedure or concept adequately. Such identification is not intended to imply recommendation or endorsement by the National Institute of Standards and Technology, nor is it intended to imply that the entities, materials or equipment are necessarily the best available for the purpose.

National Institute of Standards and Technology Special Publication 1200-17

Natl. Inst. Stand. Technol. Spec. Pub. 1200-17, 21 pages (July 2015) CODEN: NSPUE2

This publication is available free of charge from: http://dx.doi.org/10.6028/NIST.SP.1200-17 


\section{FOREWORD}

This special publication is one in a series stemming from the National Nanotechnology Initiative (NNI) Nano-EHS Research Strategy which identified Nanomaterial Measurement Infrastructure as one of the essential areas of research needed in order to develop an effective risk assessment and management plan regarding various aspects of nanotechnology in consumer products as it pertains to human health, exposure and the environment. The National Institute of Standards and Technology (NIST) was identified as a lead agency in the development of measurement strategies for the detection and characterization of nanomaterials within commercial products. Carbon nanotubes in commercial products, the focus for this special publication, were one of the nanomaterials identified in the Nano-EHS research strategy.

This guideline presents methods for the preparation and investigation of samples of multiwall carbon nanotube-polymer composites by scanning electron microscopy. It is focused on multiwall carbon nanotube epoxy composite, but the strategy should be generally applicable to other types of polymer nanocomposite materials. Updates to this protocol may be released in the future. Visit http://nist.gov/mml/np-measurement-protocols.cfm to check for revisions of this

protocol, or new protocols in the series. We also encourage users to report citations to published work in which this protocol has been applied. 


\section{Introduction}

Carbon nanotubes (CNTs) combined with polymers produce composite materials with significantly improved mechanical, electrical or thermal properties. Compared to carbon fibers CNTs offer considerable advantages, for example, it is estimated that millions of tons of kerosene fuel could be saved if CNTs were used for making the body of airplanes. The scientific and industrial interest in CNTs growing, so is global CNT production and the number of CNTcontaining consumer products [1-3]. The need to reliably detect and characterize nanocomposite materials during their lifecycle (from production to end their of existence) is also increasing. Detection and characterization of CNTs in commercial products and in the environment are difficult tasks that require sophisticated instrumentation and measurement methods. The type, surface functionalization, concentration of CNTs, and the additives used in the polymer matrix influence the efficacy of these analytical methods.

To help the detection and characterization of CNTs in nanocomposite materials, the National Institute of Standards and Technology (NIST) has developed a set of guidelines describing essential procedures for sample material fabrication, preparation, and nanomaterial investigation using several widely available measurement techniques and methods.

The procedures in these NIST guidelines focus on a simple nanocomposite system of multiwall carbon nanotubes (MWCNT) and epoxy, one of the most widely used ones. The sample preparation and measurement procedures are relevant to essentially all types of CNTs and polymers used to produce nanocomposite materials.

\section{Principles and Scope}

Scanning electron microscopy (SEM) is one of the main techniques for nanomaterial characterization, due to its ability to provide morphological details of materials at nanometer resolution. It can differentiate carbon nanotubes from other similar structures or particles with similar sizes. The highest resolution SEMs can reveal fine details of nanotube structures, including the number of carbon layers in their walls, and characterize surface coating or functionalization of tubes. With proper settings, the SEM can "see" into CNT-epoxy structures via charge-based subsurface imaging, and reveal the presence and allow for measurements of individual CNTs on the surface and inside composite materials. SEMs can also work in scanning transmission electron microscopy mode to reveal three-dimensional structure of embedded CNTs. The purpose of this special publication is to provide a detailed procedure for fabricating nanocomposite samples suitable for SEM investigations. Detailed discussion of SEM, transmitted electron microscopy (TEM) and focused ion beam (FIB) theory and operation can be found in the following references [4-7].

\section{Terminology}

Carbon nanotubes - Seamless cylinders of one or more layers (single-wall, SWCNT, or multiwall, MWCNT) of graphene with open or closed ends

Nanocomposites - Composite material formed by incorporating nanomaterial as fillers into a polymer matrix 
Scanning Electron Microscope (SEM) - Type of electron microscope that produces images of a sample by scanning it with a focused beam of electrons

Transmission Electron Microscope (TEM) - Type of electron microscope that produces images by the interaction of the electrons transmitted through the ultra-thin sample

SEM sample stub or SEM pin stub - Mounting hardware used to hold samples to be investigate in a SEM. Shape and size of a stub varies depending on the make and model of the instrument used; they are usually made of aluminum.

\section{Materials, equipment and instrumentation}

\subsection{Materials}

4.1.1 Epoxy/MWCNT composite sample

4.1.2 Double-sided conductive tape or conductive paint

4.1.3 Aluminum sample stub for mounting the nanocomposite sample

4.1.4 $3 \mathrm{~mm}$ diameter $\mathrm{Cu}$ grid for mounting thin nanocomposite sample

\subsection{Sample preparation equipment}

4.2.1 Stereo microscope for mounting the nanocomposite sample onto the sample stub

4.2.2 Sputter coater for deposition of $\mathrm{nm}$ thin osmium (Os) conductive layer

4.2.3 Tweezers

4.2.4 Razor blade

4.2.5 Latex or nitrile gloves

4.2.6 Personal protection devices

\subsection{Instrumentation}

4.3.1 High-resolution scanning electron microscope

\section{Sample Preparation for SEM investigation of CNT nanocomposites}

\subsection{Sample size and geometry}

Concentration of CNTs in commercial nanocomposites is relatively low $(<5$ wt. \%) and many CNT nanocomposites are poor electrical conductors unless they are specifically engineered to be conductive. To prevent excessive charging of the specimen under electron beam investigation, it is recommended that the sample size be minimized $(5 \mathrm{~mm}$ or less in lateral dimensions).

\subsection{Sample preparation}

A small piece of material can be readily cut from the bulk material using a razor blade or a microtome machine. Additionally, having a relatively flat and planar geometry (e.g. small piece 
of nanocomposite film) will facilitate easier specimen preparation and measurement. Another method for preparing cross sections of CNT-nanocomposites is cryo-fracturing. Carefully immersing the sample into liquid nitrogen and handling it with protective gloves, one can shatter it into small pieces. Pairing the fractured parts makes investigation on two sides of the fractured location possible. Cryo-fracturing generally results in less distortion than the use of shear forces and room temperature fracturing.

\subsection{Bulk sample mounting}

Sample mounting hardware/stub varies depending on the type of SEM used. The preferred method to secure the small piece of nanocomposite is the use of a couple thin phosphor bronze springs and small-size screws that apply only small forces. In this case there is no need for adding a conductive layer at the bottom. Alternatively, the sample can either be attached to the stub using a double-sided conductive tape (e.g., carbon tape or copper tape) or using a conductive paint such as carbon paint or silver paint. If using a conductive paint, use small amount of paint to prevent the paint from wicking over the sample. In these cases one should wait for a longer period of time (up to an hour) for the conductive tape to "settle" or for the paint to completely dry, otherwise, the sample could drift and/or get contaminated by the outgassing solvent. See Figure 1 for a schematic illustration of mounting of a bulk nanocomposite sample.

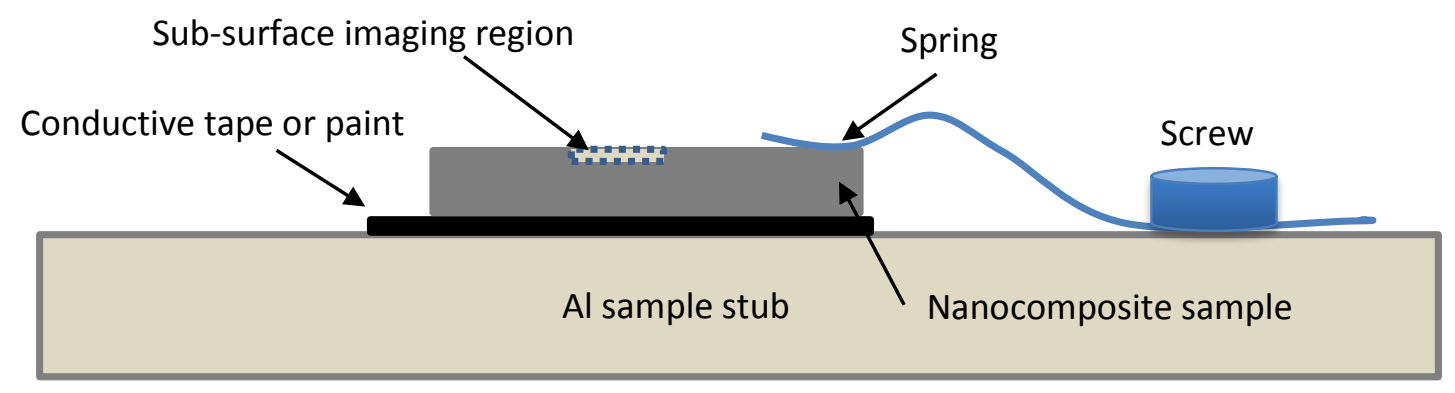

Figure 1. Sample mounting using an Al stub; the preferred method (spring and screw) is depicted on the left, in which case the conductive tape or paint is omitted

\subsection{Thin slice sample mounting on TEM holders}

For STEM imaging and measurements in the SEM CNT-epoxy samples must be prepared by cutting about $100 \mathrm{~nm}$ thin slices for the bulk sample. This can be done with a microtome machine. The slices must be sufficiently electron transparent at the landing energies used. On approximately $100 \mathrm{~nm}$ thin CNT-epoxy samples even $5 \mathrm{keV}$ electron beam could give useful images, so some experimentation is required to arrive at the optimal thickness and landing energy of the SEM. The thin slices should be mounted on TEM standard $3 \mathrm{~mm} \mathrm{Cu}$ grids or other special TEM sample holders. The thin slice readily adheres to the surface of the $\mathrm{Cu}$ grid, so generally no other treatment or adhesive is required. It is important to prevent the layers from overlapping. The goal is to have several windows covered by a single layer of the thin slice. Figure 2 shows an example of $\mathrm{Cu}$ grid-mounted thin MWCNT-epoxy sample. The slice is dark over the brighter $\mathrm{Cu}$ surface. 
It is advantageous to use a very sharp knife to avoid rippled sample surface, e.g. stripes left behind by the uneven edge of the blade microtome machine. Conductive coating of the mounted thin nanocomposite sample is not necessary if $\mathrm{Cu}$ or other conductive grid is used.

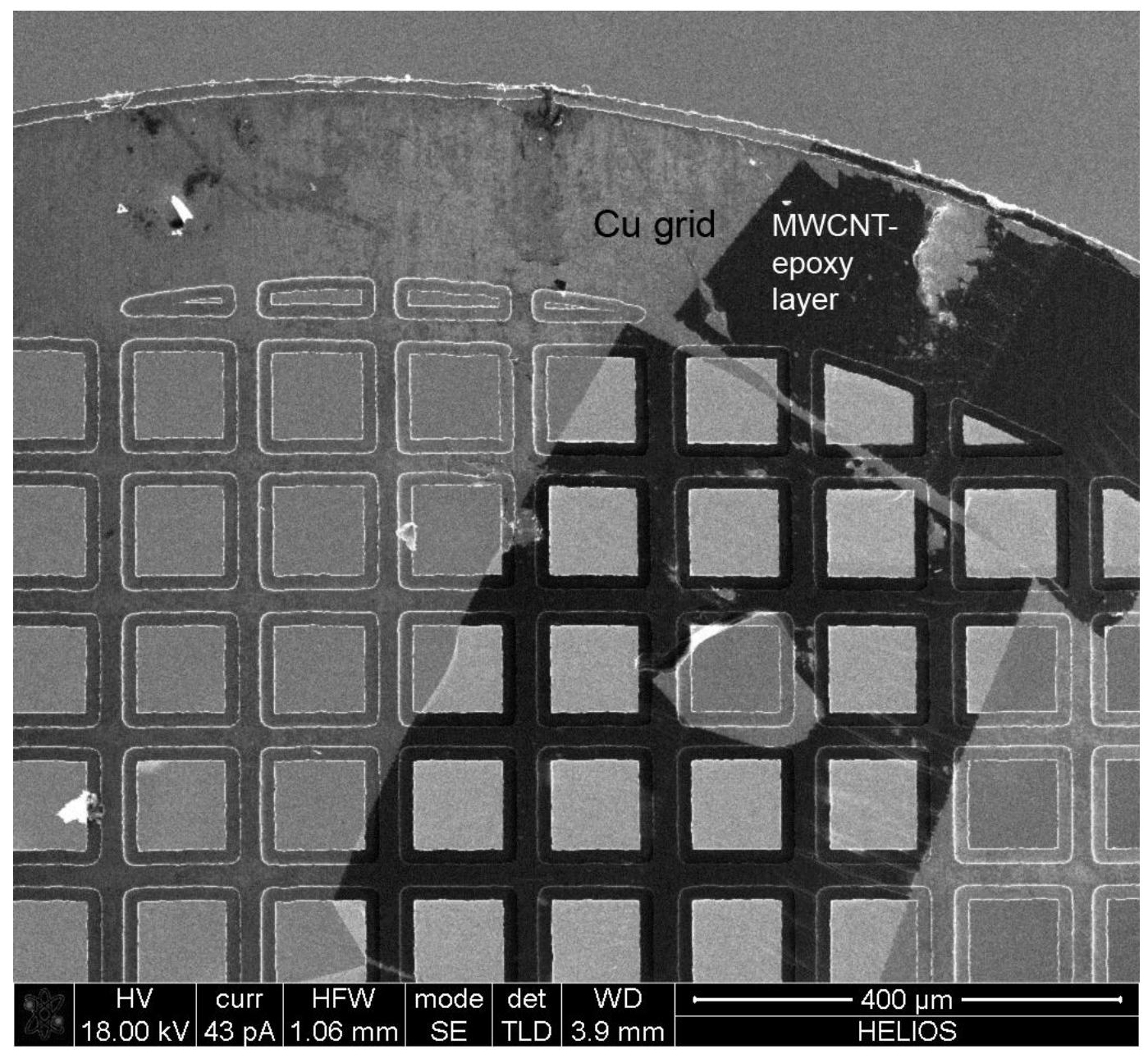

Figure 2. Thin slice of MWCNT-epoxy sample mounted on Cu TEM sample holder

\subsection{Sample coating}

Coating the mounted nanocomposite sample with a thin layer of conductive material is not recommended for charge-based imaging, as it will defeat it. For high-resolution imaging of individual or set of CNTs special osmium (Os) coating is recommended [8]. With osmium coaters one can achieve conductivity at a thickness of slightly less than $1 \mathrm{~nm}$, but in some cases a somewhat thicker layer might be needed for completely charge-free imaging of CNT-epoxy composites.

Traditional coatings, such as $\mathrm{Au}, \mathrm{Au} / \mathrm{Pd}$, Pt, etc. are not recommended, because these layers are generally too thick and develop disturbing structures over the surface of the sample. In some cases samples coated this way could still reveal useful information, but uncertain measurement errors with small-diameter CNTs with an unknown, possibly several nm thick metal layer around them are unavoidable. 


\section{SEM imaging}

CNT-nanocomposites are not conductive enough to allow for imaging that is completely free of sample charging, but -except for very low $(<0.01 \mathrm{wt} \%$ ) CNT concentrations- might be conductive enough for charge-based SEM imaging. This type of SEM imaging is unique to CNT-nanocomposites. It is governed by a set of complex charging phenomena, and in many cases makes it possible to "see" into the sample, in some instances down to several hundreds of nanometer depths [9]. It is generally agreed that the contrast of subsurface CNTs arises from changes in secondary electron (SE) yield due to charge contrast [10]. Due to the shallow escape depth of SEs, direct SE emission from CNTs much beyond about $7 \mathrm{~nm}$ to $10 \mathrm{~nm}$ depths should not be detectable [6]. Charge contrast of CNT-nanocomposites is still not a completely settled matter; there are several explanations for it at the various landing energies SEM can be used for the investigation of CNT-nanocomposites [10].

Three-dimensional charge-contrast imaging is also possible, and good quality information can be gained about the sub-surface distribution of CNTs. Charge-based imaging is time- and dose-dependent, and depending on the particular CNT-nanocomposites may require some experimentation to find out the best pre-charging and imaging landing energies (accelerating voltages). Simply changing the settings of the SEM one may get images that show two different types of charge-based images. Positive charging is more common, on these SEM images that areas that have less CNT are brighter, in negative charging darker than the rest of the sample.

One $\mathrm{nm}$ or so thin metal coating of the surface of CNT-nanocomposites makes highresolution imaging at 100000 times or higher magnifications feasible. The thin metal, preferably Os coating allows for the assessment of the size and distribution of CNT-rich areas, and for the establishment of the correlation between coated and uncoated, charged-based images, which may eliminate the need for metal coating of samples in high throughput measurements of CNT loading and distribution in CNT-nanocomposites.

\subsection{Charge-based SEM imaging}

SEM images of uncoated CNT-nanocomposites may look very different depending on the landing energy (accelerating voltage) used for their imaging. Figure 3 shows a 5 wt. \% MWCNT CNT-nanocomposite sample imaged with $0.3,1,2,3,15$ and $30 \mathrm{keV}$ electron landing energy. These images were taken on the surface of the sample; contrast reversal for the $1 \mathrm{keV}$ image turns some areas darker or brighter, depending on the local state of the sample charging on the surface and deep in the sample. The higher landing energy images show areas that are CNT-rich darker than the bright epoxy-rich areas.

Figure 4 shows a $5 \mathrm{wt}$ \% MWCNT CNT-nanocomposite sample imaged with 15, 10, 5, 3, 2 and $0.8 \mathrm{keV}$ electron landing energy. The secondary electron images of the same area of the cross section show varying contrast, even slight contrast reversal for the $0.8 \mathrm{keV}$ image on the far right. Lager energies ( $2 \mathrm{keV}$ and up) show embedded CNT-rich areas as dark spots, and the CNTs that come to the surface and stand above it also vary in their appearance. These contrast differences are due to the varying landing energy, i.e., the extent of the penetration of the electron beam, and a complex internal system of conductive and non-conductive areas around the CNTs. 

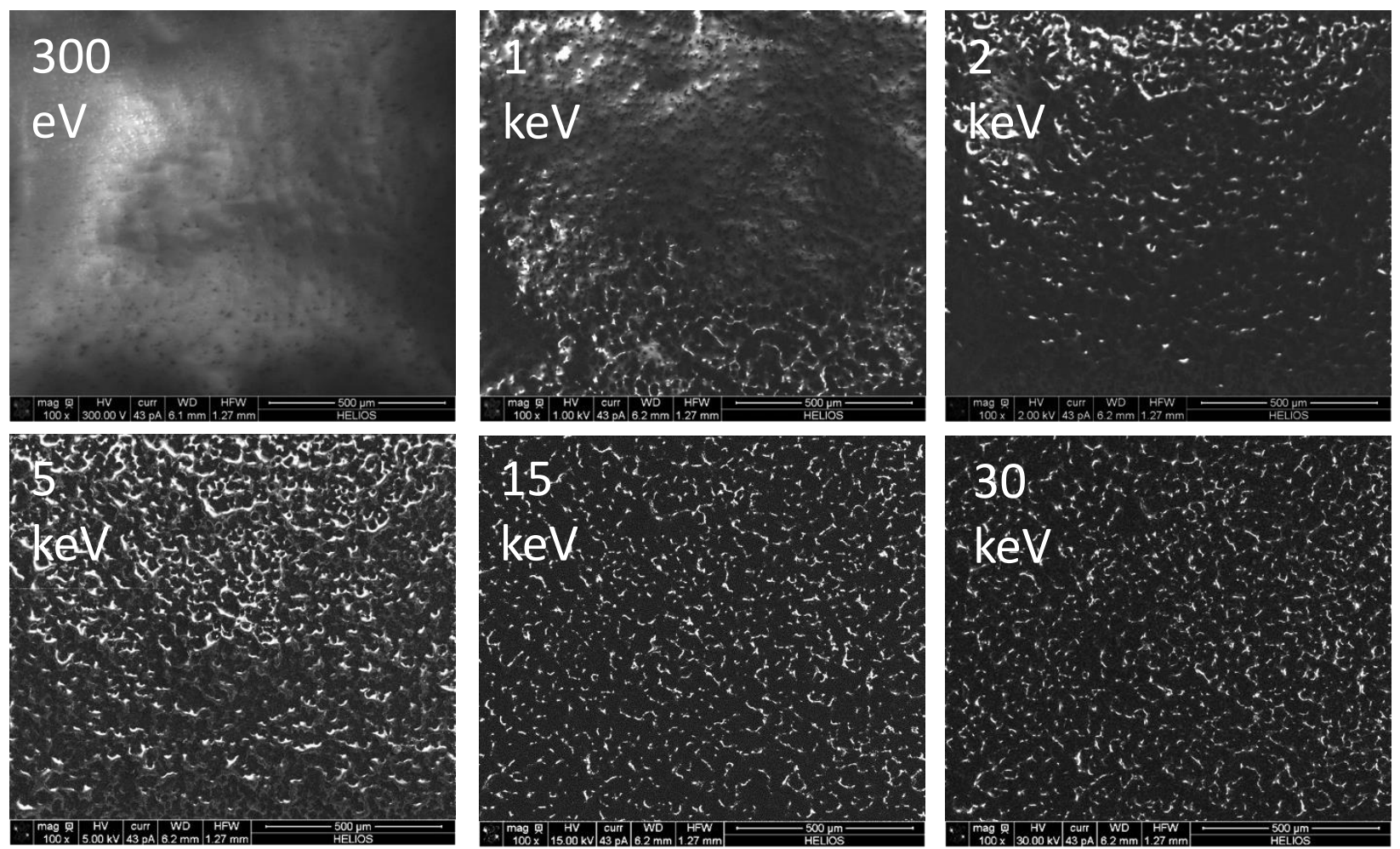

Figure 3. Charge-based SEM images taken on the top of a 5 wt. \% MWCNT CNTnanocomposite sample with various electron landing energies, $1.27 \mathrm{~mm} \mathrm{HFV}$

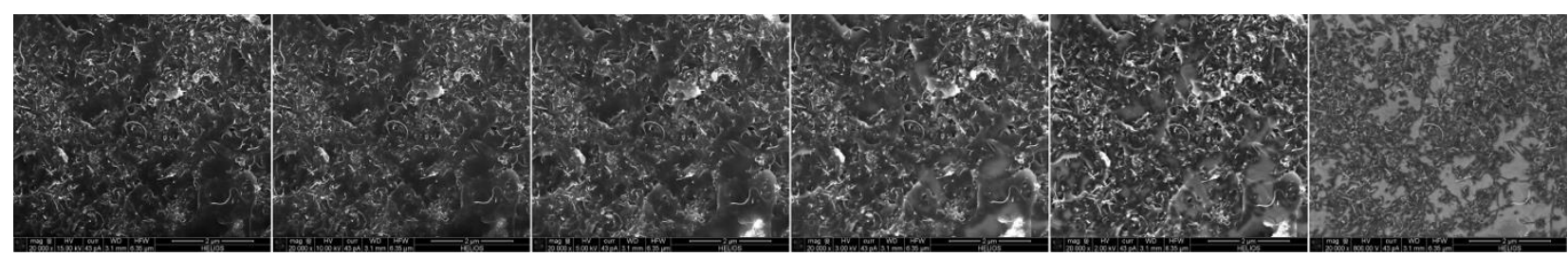

Figure 4. Charge-based SEM images taken on a $5 \mathrm{wt}$ \% MWCNT CNT-nanocomposite sample with $15 \mathrm{keV}$ (leftmost), 10, 5, 3, 2 \& $0.8 \mathrm{keV}$ (rightmost) electron landing energy, $6.35 \mu \mathrm{m} \mathrm{HFV}$

Charge-based SEM imaging of CNT-nanocomposite samples is a time-dependent phenomenon, reaching a stable state may requires pre-charging of the sample. Figure 5 illustrates the gradual charge-up of a $1 \%$ wt. MWCNT-nanocomposite sample. These are tilted images to show the side also and the flat top. They show gradual charge build-up and the stable patterns of the charged epoxy-rich and MWCNT-rich areas. The top patterns are bright as they reached their stable charging state fast, and remains essentially unchanged. The exposed left wall of the sample charges up at a slower rate due to its weaker dose of electrons; the gradual stabilization of the patterns is observable there also. 


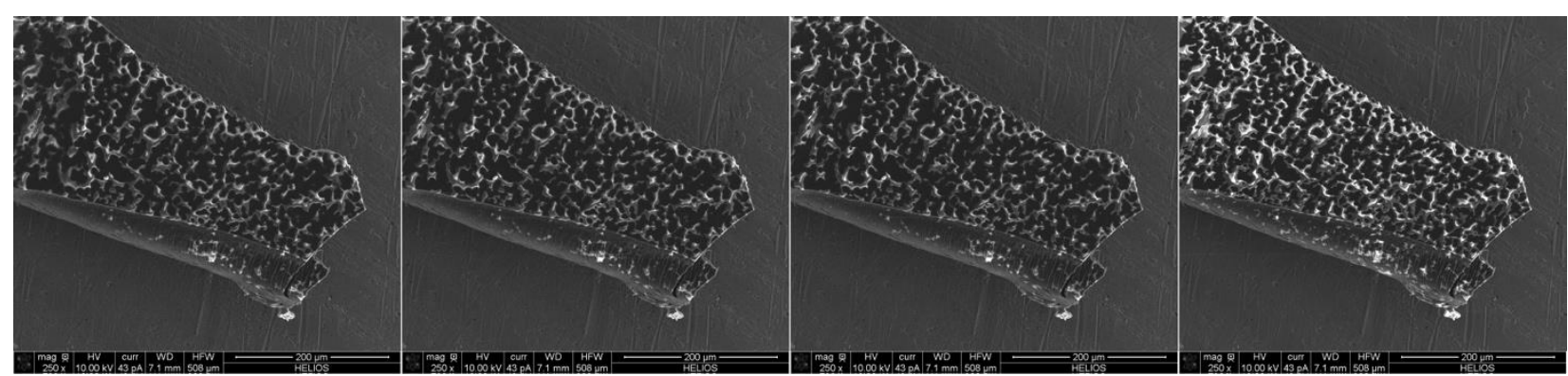

Figure 5. Fast (on top) and slow (at the side) gradual charge-up of a $1 \%$ wt. MWCNTnanocomposite sample. $608 \mu \mathrm{m} \mathrm{HFV}$

Figure 6 shows how charge contrast can reveal the MWCNT- and epoxy-rich areas. Tilted, flat and cross-sectional $1 \mathrm{wt}$. \% MWCNT-nanocomposite sample images show that charge build-up yields patterns of brighter epoxy-rich and darker MWCNT-rich areas. The image on right was taken immediately after the left one, which essentially got double dose of electrons.
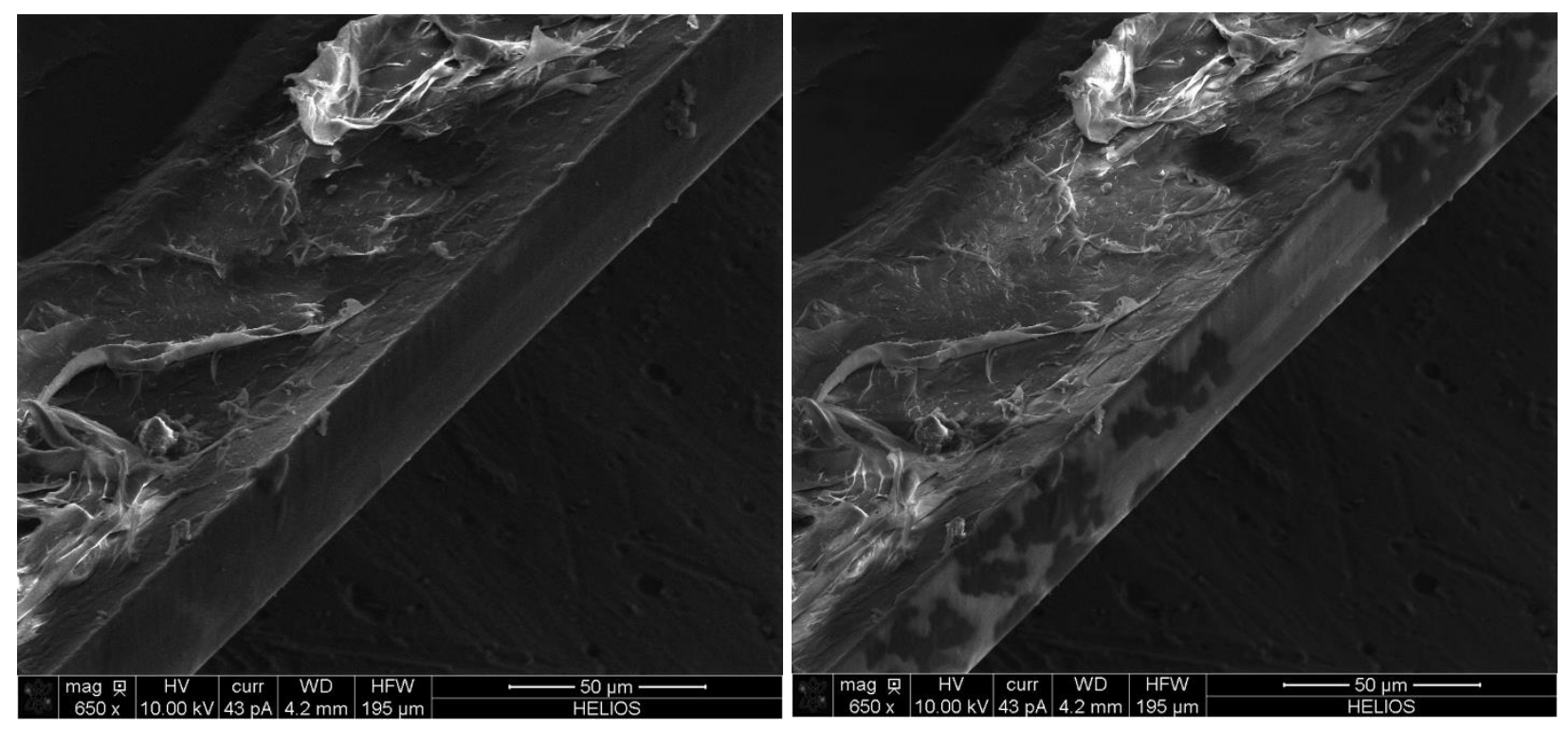

Figure 6. Charge contrast reveals the MWCNT- and epoxy-rich areas. Charge build-up yields patterns of brighter epoxy-rich and darker MWCNT-rich areas. The image on right was taken immediately after the left one. $195 \mu \mathrm{m} \mathrm{HFV}$

Figure 7 shows the unique phenomenon of charge-contrast that allows for "seeing" into the bulk material.

In this instance the SEM images taken on a $1 \mathrm{wt}$ \% MWCNT-epoxy nanocomposite sample show that the CNTs are mostly in small-size clumps with uneven distribution. The brightest ones are closer to the surface, and there are a fewer of these there than deeper in. 


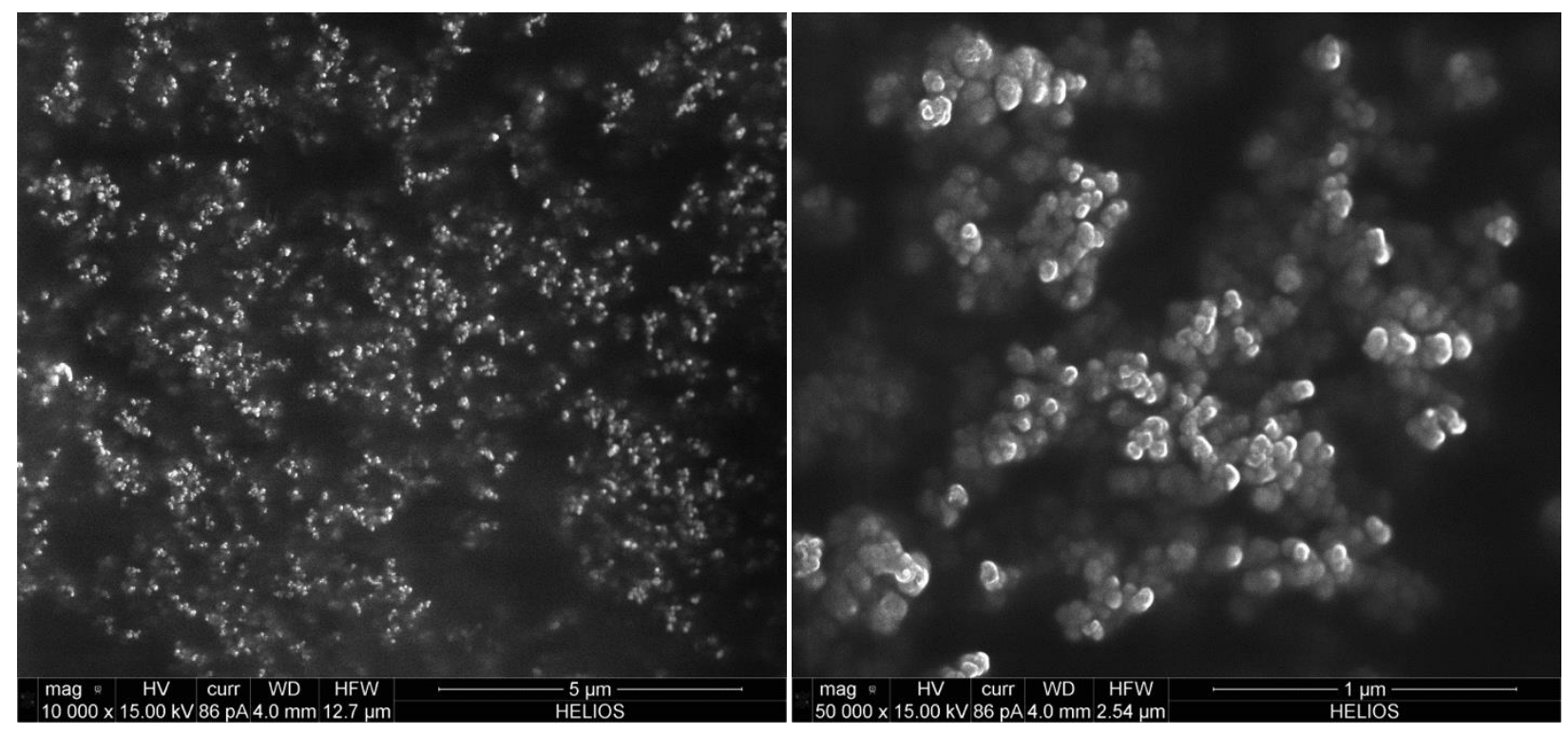

Figure 7. Charge-contrast of small-size MWCNT clumps with uneven distribution in bulk MWCNT-epoxy sample. There are fewer of these closer to the surface, than deeper in. $2.54 \mu \mathrm{m}$ HFV

\subsection{Stereo sub-surface charge-based SEM imaging}

Taking charge-based SEM images from different angles allow for the three-dimensional (3D) imaging and measurements of the CNT distribution at moderately high magnifications. As an example, Figure 8 on the top shows an image pair of $0.5 \mathrm{wt} \%$ SWCNT-epoxy sample tilted at $-5^{\circ}$ (left) and $+5^{\circ}$ (right) angles. On the bottom is a 3D SEM image generated with these. The red arrows point to a CNT very close to the surface, the blue ones point to a CNT embedded at an estimated depth of $260 \mathrm{~nm}$. [10] 

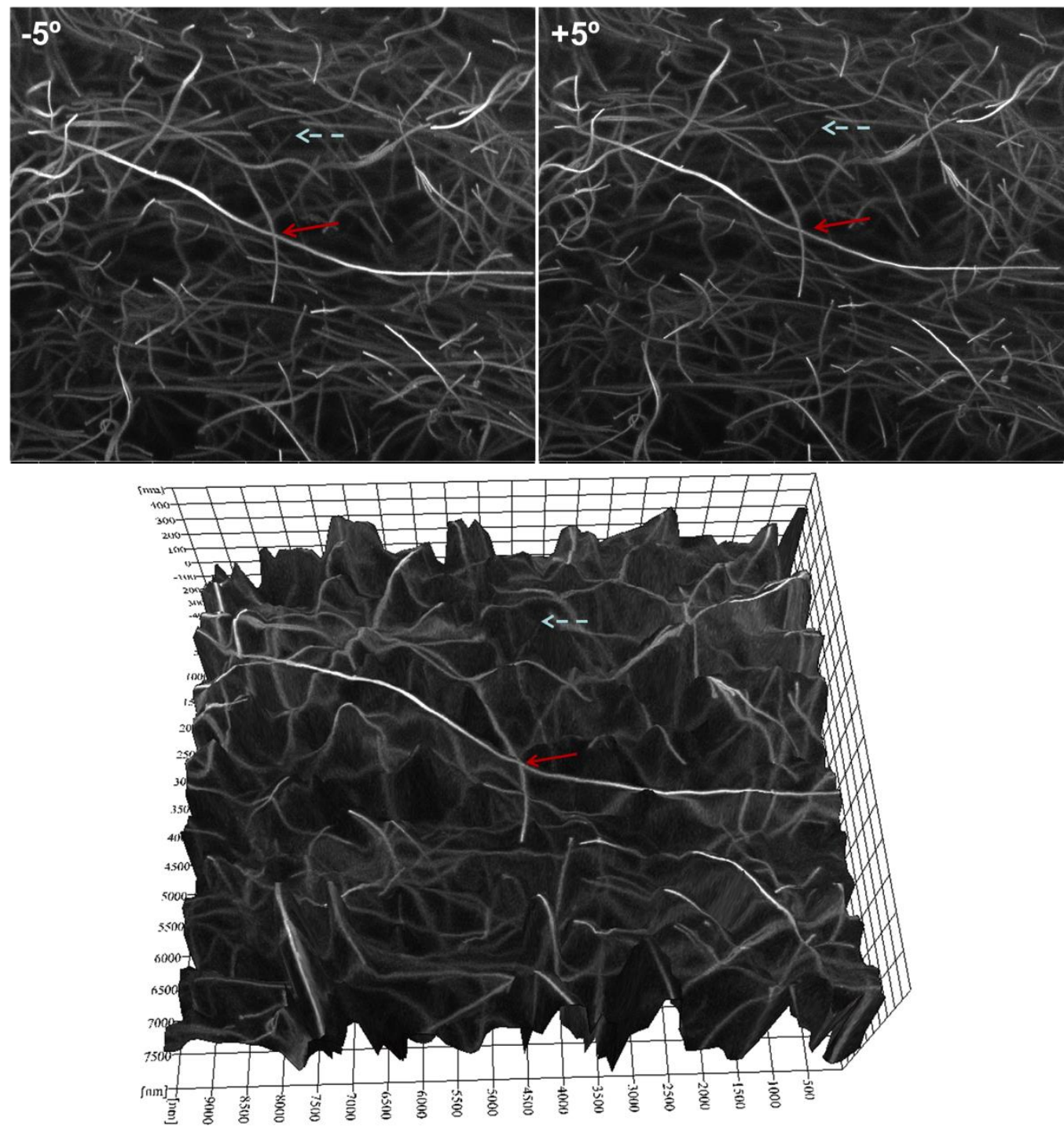

Figure 8. Top: $28 \mathrm{kV} \mathrm{SEM} \mathrm{image} \mathrm{pair} \mathrm{of} \mathrm{a} 0.5 \%$ SWCNT-epoxy sample tilted at $-5^{\circ}$ (left) and at $-5^{\circ}$ (right) angles, $10.6 \mu \mathrm{m}$ HFV. Bottom: 3D SEM image generated with these images. The red arrows point to a CNT very close to the surface, the blue ones to a CNT embedded at an estimated depth of $260 \mathrm{~nm}$.

\subsection{SEM imaging of non-charging samples}

A couple of nanometer thin layer of metal can eliminate strong sample surface charging by draining excess electrons to ground, and keeping the sample surface potential at the same potential as the ground. It does not, cannot eliminate charging inside the sample, so some charge contrast within the bulk of the sample can still be observed. Figure 9 shows a cryo-fractured 1 wt. \% MWCNT-nanocomposite sample at locations where the clumped up CNTs broke in half, and individual CNTs are on the surface. Thin Os coating was applied to the sample, which 
allows for charging artifact-free imaging at high magnification. The energetic electron beam penetrates the thin CNTs and produces higher SE yields where they cross. This can be observed especially well on the left where several CNTs are protruding over a darker area. The image on the right confirms that there are individual CNTs also, not just those that clumped up.
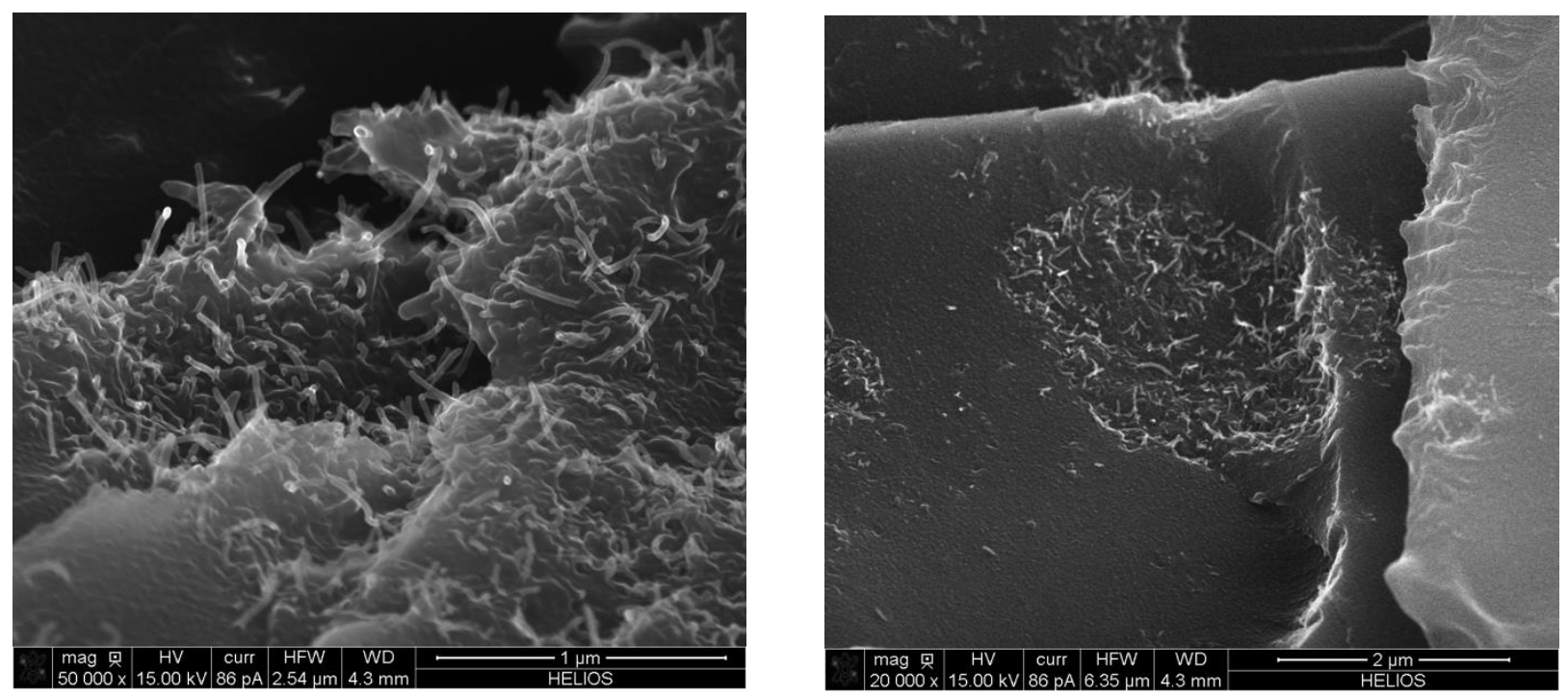

Figure 9. Cryo-fractured 1 wt. \% MWCNT-nanocomposite sample with thin Os coating. Left: the energetic electron beam penetrates the thin CNTs and produces higher SE yields where they cross. The right image confirms that there are individual CNTs also. $6.35 \mu \mathrm{m} \mathrm{HFV}$

Figure 10 shows the top of the $2 \mathrm{~nm}$ Os-coated sample of Figure 4 with darker areas clearly discernable where $1 \mathrm{wt}$. \% MWCNTs clumped into $5 \mu \mathrm{m}$ to $10 \mu \mathrm{m}$-size globulae. Fine details of the sample surface are also clearly visible. The sample charges inside to different charging states at the CNT-rich and the epoxy-rich areas, and the charge difference leads to different SE yields.

A comparison of Os-coated and non-coated areas indicates that CNT loading and distribution in MWCNT-nanocomposite samples can be estimated without sample coating. Figure 11 shows the tip of the sample shown on Figure 5 before Os coating on the left and after coating on the right. The bright areas on the non-coated samples are epoxy-rich, while the dark areas are CNT rich, there carbon nanotubes limit sample charging and SE emission. The Oscoated image reveals further fine details and also structures on the top surface that cannot be seen otherwise. 


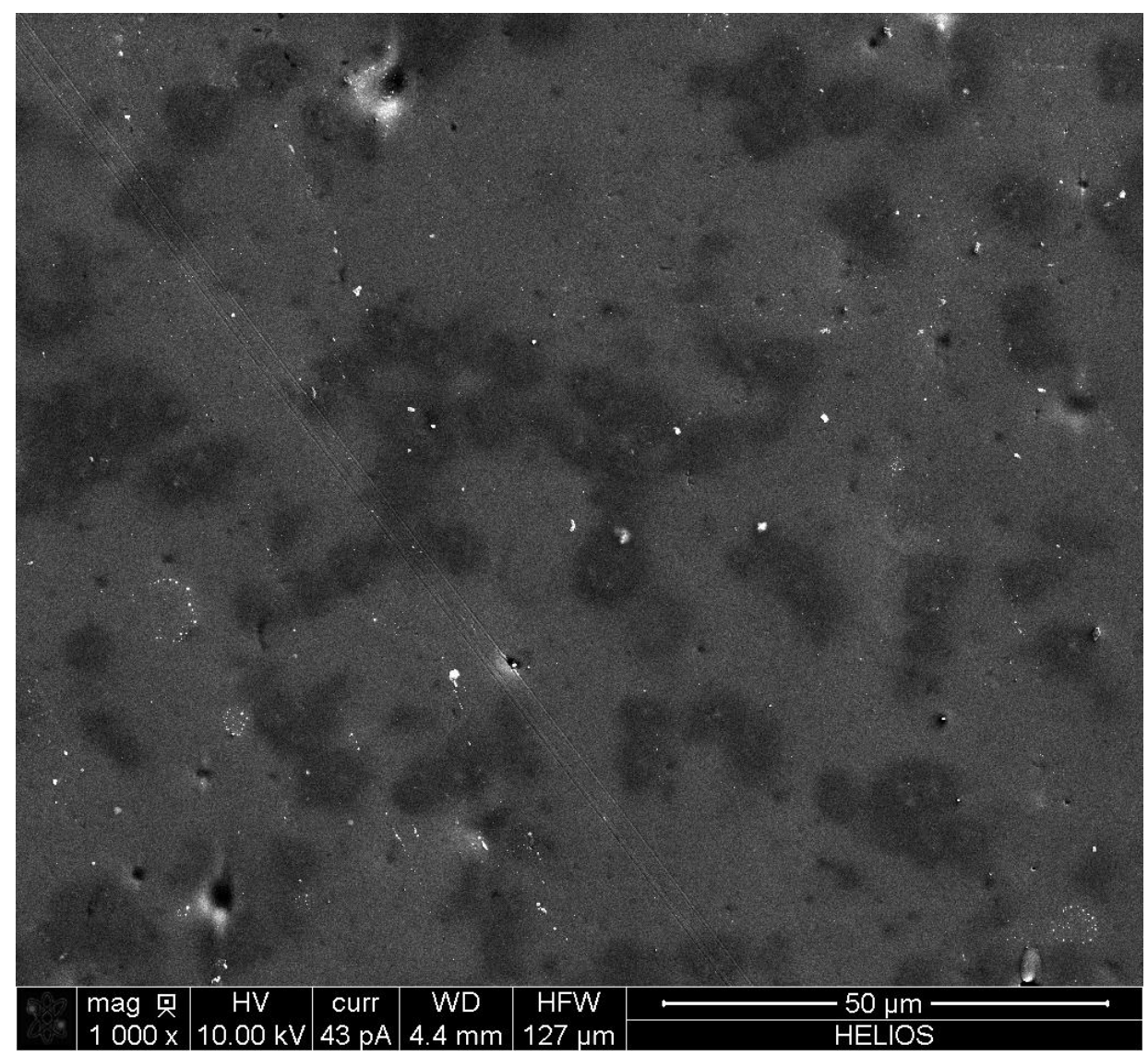

Figure 10. Sample of Figure 5 after $2 \mathrm{~nm}$ Os coating. The dark areas are CNT rich. $127 \mu \mathrm{m} \mathrm{HFV}$
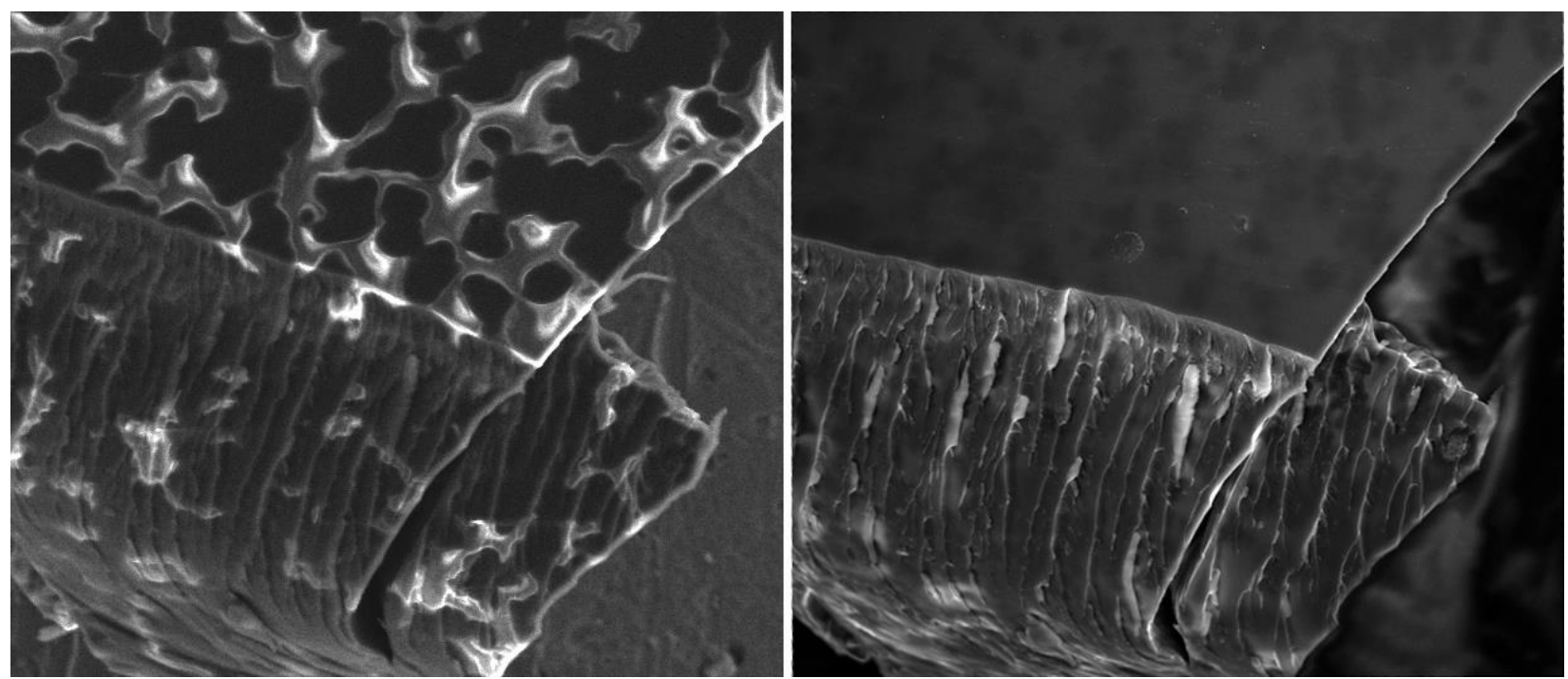

Figure 11. The sample of Figure 5 before Os coating (left) and after (right). The bright areas on the non-coated samples are rich in epoxy, the dark ones are in CNT. $50.8 \mu \mathrm{m} \mathrm{HFV}$ 


\subsection{SEM imaging in transmitted electron modes}

Slicing the MWCNT-epoxy samples into about 100 nanometer thin layers offers the possibility of investigating the sample in transmitted electron imaging mode. Dedicated TEM/STEM instruments have better resolution, but it is easier to work with the SEM in STEM mode, and even at $10 \mathrm{keV}$ landing energy sufficient transmission might be possible. Bright-field (BF), dark-field (DF) and high-angle annular dark-field (HAADF) STEM imaging certainly adds useful information to the secondary electron (SE) mode imaging. The contrast of these imaging modes may vary substantially, so experimentation is necessary. The signals coming from very small volumes are inherently weak, especially because high primary electron beam currents can ruin the sample. To achieve sufficiently noise-free image, several minute-long acquisition times are typical. SE imaging mode offers more surface-related information, while the various TEM modes provide information on the whole cross section of the sample. DF and HAADF modes images use (highly) scattered transmitted electrons, and may reveal the presence of otherwise not perceptible structures. Figure 12 shows the SE and HAADF images of the same sample, which were acquired at simultaneously.

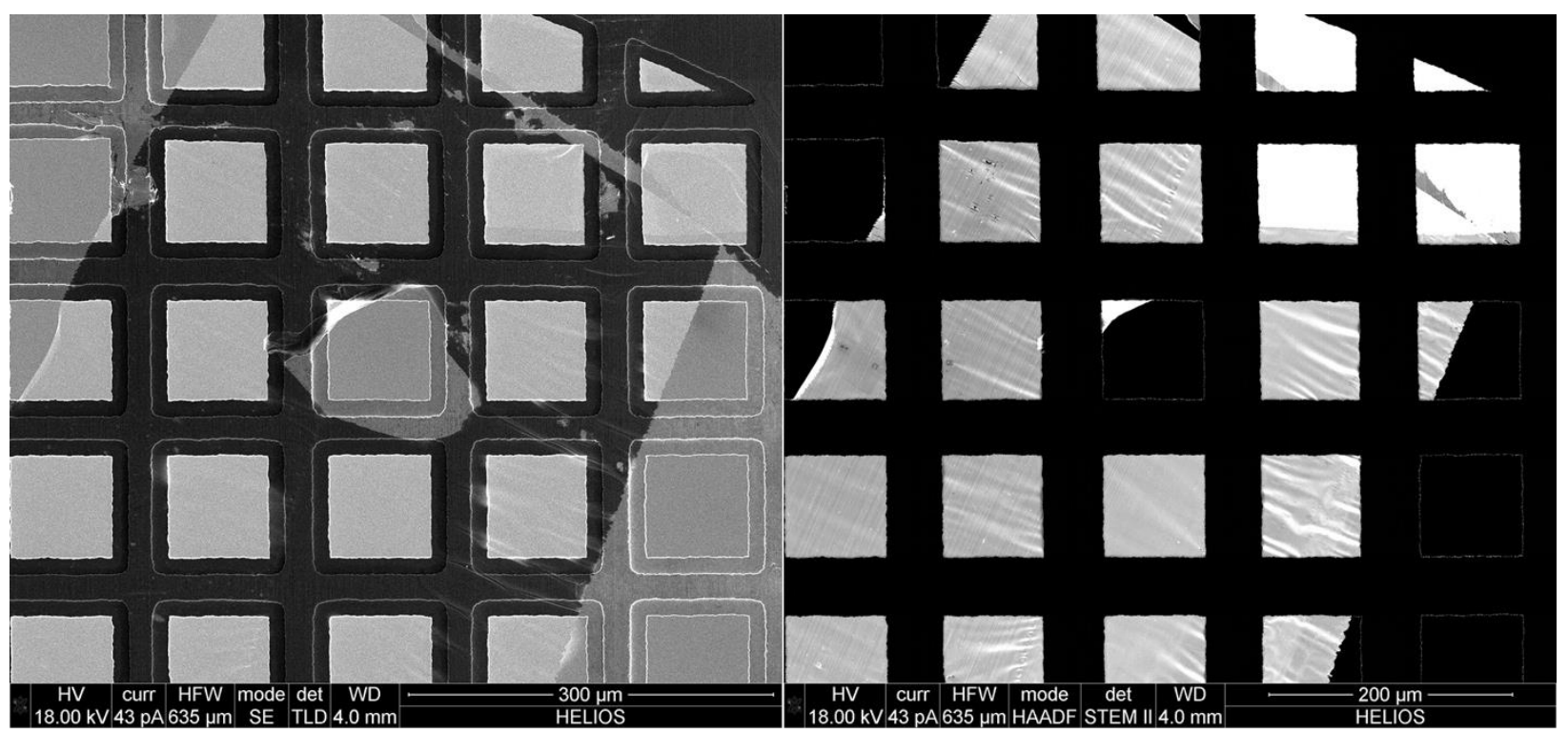

Figure 12. Simultaneously acquired SE (left) and HAADF SEM (right) images of a thin slice of an MWCNT-epoxy sample. Note the different contrast. $635 \mu \mathrm{m}$ HFV

In many cases the $\mathrm{BF}$ and $\mathrm{DF}$ images are inverses one another, but subtle differences can be seen and it might be easier to detect some sample feature in one or in the other. Figure 13 shows a high-resolution image pair of a 1\% MWCNT sample. CNT rich areas and individual CNTs are clearly visible, and the wall of some of the CNTs as well. To some extent the threedimensionality of the CNTs can be ascertained, CNTs give stronger or weaker contrast as they curve up or down. In some cases some embedded material may show up within the CNTs. It is important to corroborate these information to ensure proper interpretation of the SEM/STEM images, possibly with dedicated TEM/STEM investigation, or with other techniques. 


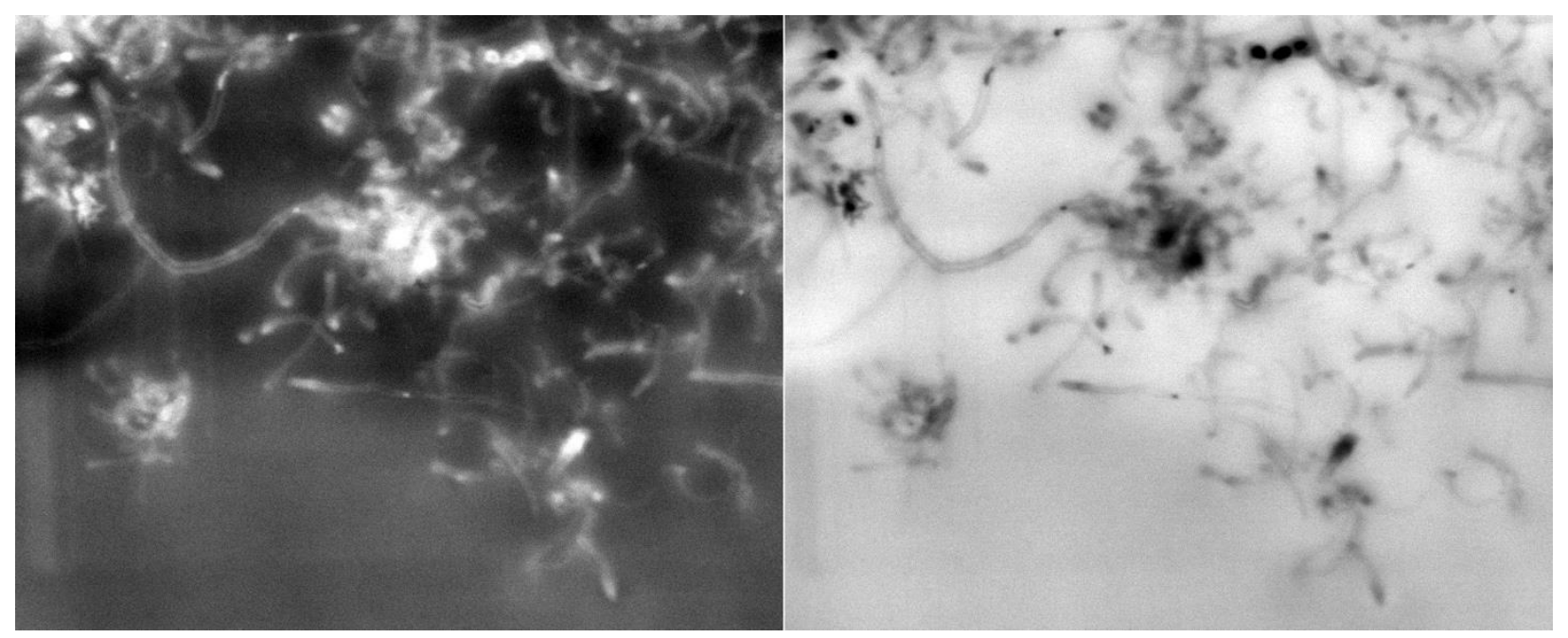

Figure 13. DF (left) and BF (right) STEM images of a thin slice of a $1 \%$ MWCNT-epoxy sample. $1.21 \mu \mathrm{m} \mathrm{HFV} \mathrm{for} \mathrm{both.}$

HAADF imaging could prove to be the most sensitive to reveal the distribution of CNTs in thin slices of CNT-epoxy samples. Figure 14 shows an HAADF image of a 1\% MWCNT-epoxy sample. The MWCNT distribution is typical; there are a lot of CNTs in large quantities together, and some dispersed across the field of view. The darker stripes (angled at about 28 degrees) are coming from the microtome knife, due its not perfectly sharp edge, the top and the bottom of the slice are not completely even, and give some contrast.

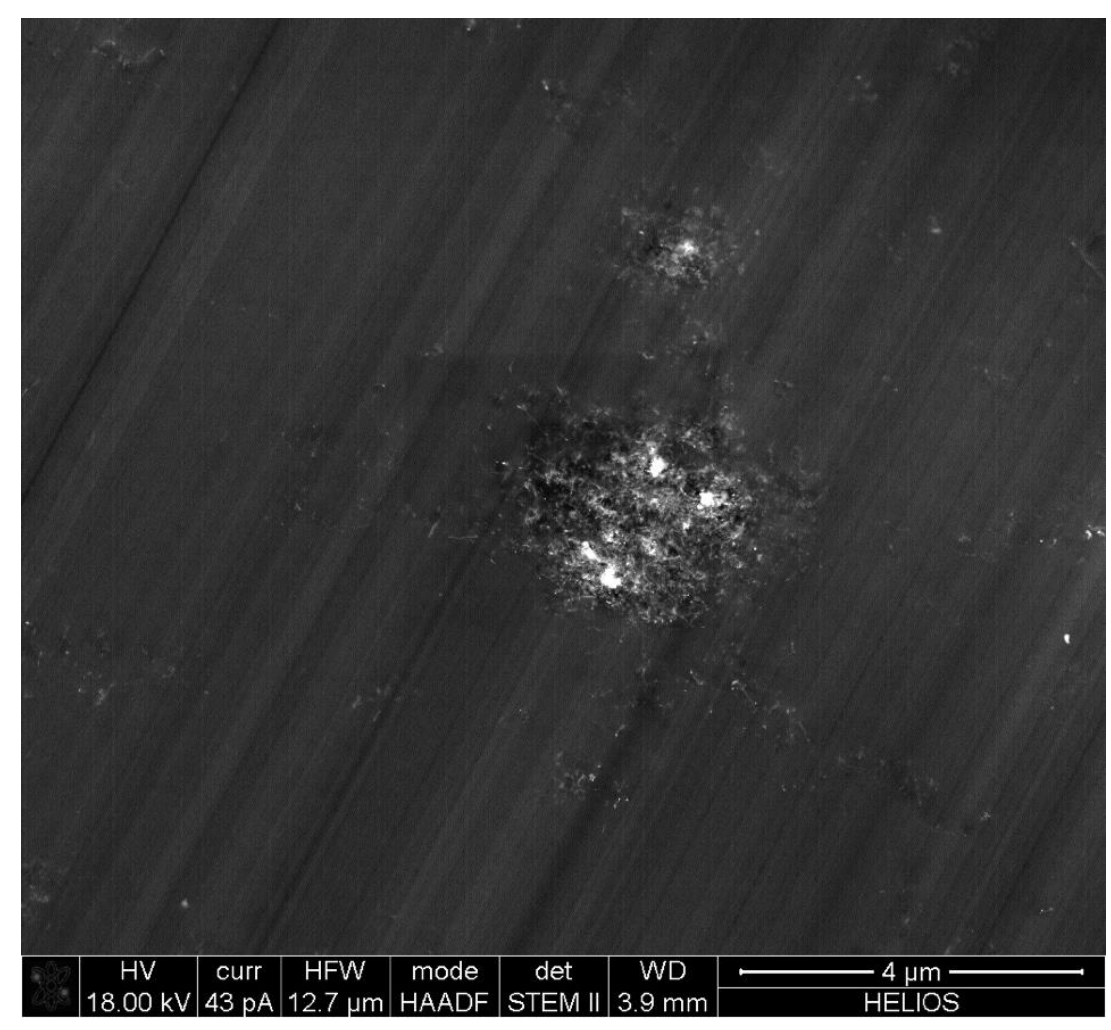


Figure 14. HAADF STEM image of a thin slice of a 1\% MWCNT-epoxy sample. $12.7 \mu \mathrm{m} \mathrm{HFV}$ 6.5 Stereo sub-surface SEM imaging in transmitted electron mode

Slicing the MWCNT-epoxy samples into about 100 nanometer thin layers offers another possibility: the investigation of the sample in transmitted electron imaging mode to reveal the 3D distribution of MWCNTs at high magnifications. At these magnifications the individual CNTs are well visible, so are the catalytic and other materials. Figure 15 shows a BF STEM image pair of a thin slice of a $1 \%$ MWCNT-epoxy sample. The left image was taken at $-5^{\circ}$, the right one at $-5^{\circ}$ tilt. The 2560 horizontal pixel resolution allows for seeing various MWCNTs with varying number of walls, and catalytic residues as small as $3 \mathrm{~nm}$ or so adhering to the walls. The CNTs turn, curl up and intertwine, the ones that are standing close to parallel to the direction of the primary electron beam (perpendicular to the image), turn dark as there are more carbon atoms that stand in the way of the electron beam, causing a dark contrast. The epoxy surrounds the CNTs almost completely uniformly, but there are a few locations where the epoxy appears uneven.

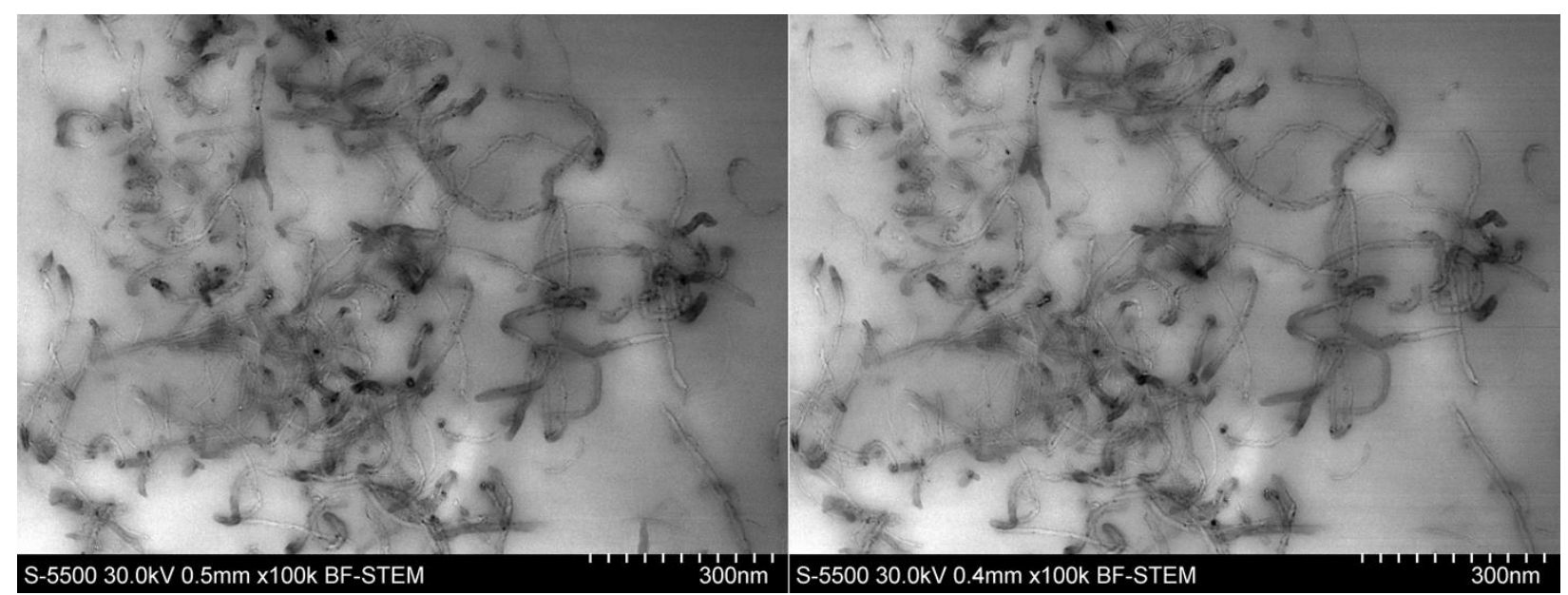

Figure 15. BF STEM image pair of a thin slice of a $1 \%$ MWCNT-epoxy sample. The left image was taken at $-5^{\circ}$, the right one at $+5^{\circ}$ tilt. $1.587 \mu \mathrm{m} \mathrm{HFV}$

All these are better perceptible by the human vision in 3D. Figure 16 shows a red-cyan color-coded 3D representation of the MWCNT sample generated by using a portion of the DF STEM image pair of Figure 15. To see the scene in 3D, red-cyan glasses are necessary. The image portions of the right half of the images were turned counter-clockwise, i.e., $-90^{\circ}$ to align them properly for 3D viewing in one of the suitable several software packages [11], which can perform the steps necessary to turn a stereo image pair into color-coded 3D image. The 3D image reveals more information out of the information present in the two images, than the image pair without 3D visualization. It may take a little time for the eyes and the brain to truly see things in 3D with the red-cyan glasses, but once they do, the scene is amazing. 


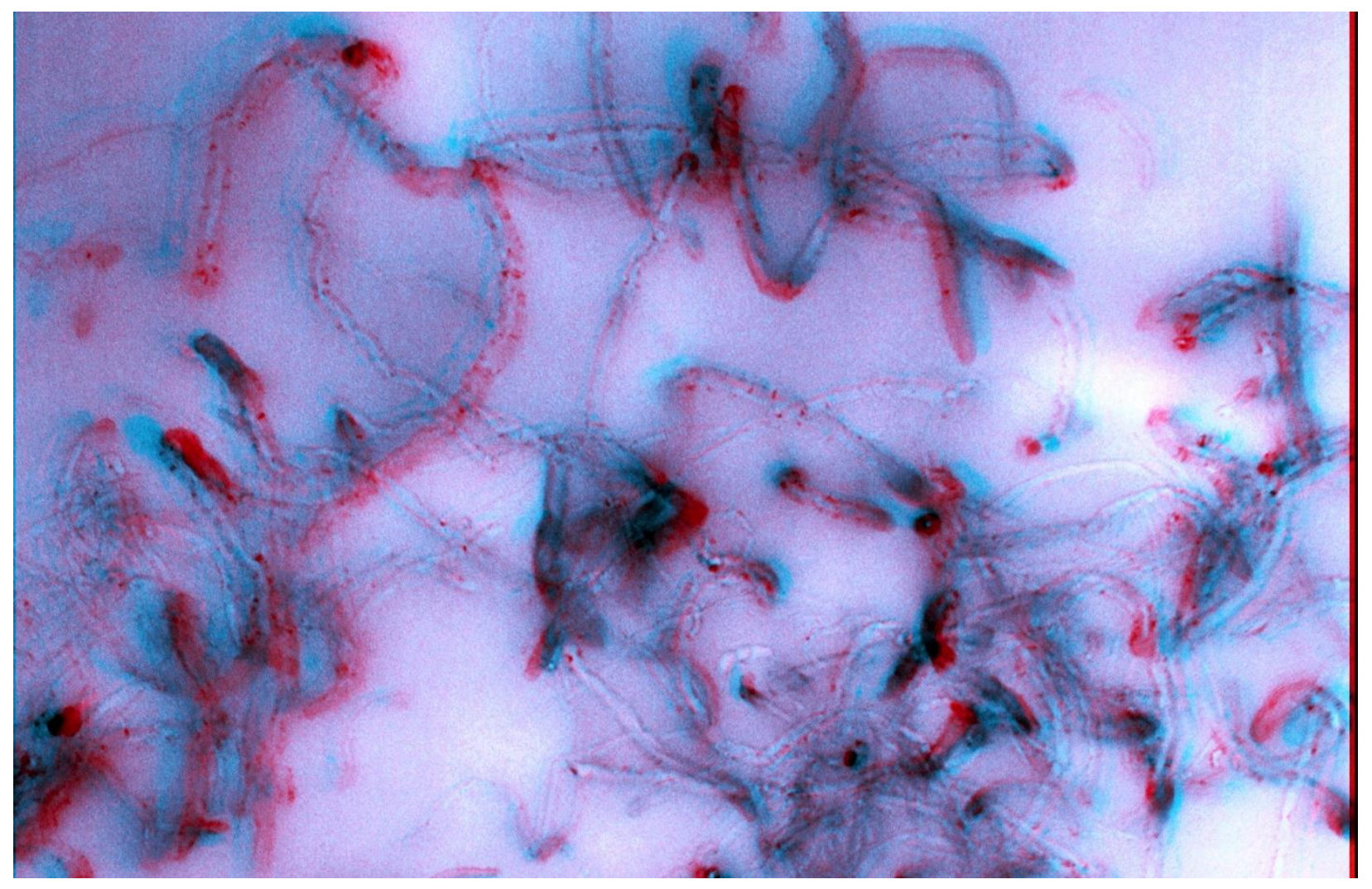

Figure 16. Red (right) - cyan (left) coded 3D representation of the MWCNT sample generated using a portion of the DF STEM image pair of Figure 15. To see the scene in 3D, red-cyan glasses are necessary. $1.11 \mu \mathrm{m} \mathrm{HFV}$

\section{Abbreviations}

$\begin{array}{ll}\text { CNT } & \text { carbon nanotube } \\ \text { SWCNT } & \text { single-walled carbon nanotube } \\ \text { MWCNT } & \text { multi-walled carbon nanotube } \\ \text { SEM } & \text { scanning electron microscopy } \\ \text { TEM } & \text { transmission electron microscopy } \\ \text { keV } & \text { kilo electron volt } \\ \text { HFV } & \text { horizontal field of view } \\ \text { HFW } & \text { horizontal field width } \\ \text { BF STEM } & \text { bright-field transmission electron microscopy } \\ \text { DF STEM } & \text { dark-field transmission electron microscopy } \\ \text { HAADF STEM } & \text { high-angle annular dark-field transmission electron microscopy }\end{array}$

\section{Acknowledgements}


I would like to thank Debra Kaiser for her direction and vision in the characterization of nanomaterials in consumer products, from which the development of this protocol derived.

\section{References}

1. National Nanotechnology Initiative: Environmental, Health and Safety Research Strategy. 2012, Presidents Council of Advisors on Science and Technology: National Science and Technology Council Committee on Technology: Washington, DC.

2. De Volder, M.F.L., Tawfick, S.H., Baughman, R.H. \& Hart, A.J. (2013) "Carbon Nanotubes: Present and Future Commercial Applications”, Science, 339, 535-539 doi: http://dx.doi.org/10.1126/science.1222453

3. Kingston, C., Zepp, R., Andrady, A., Boverhof, D., Fehir, R., Hawkins, D., Roberts, J., Sayre, P., Shelton, B., Sultan, Y., Vejins, V. \& Wohlleben, W. (2014) "Release characteristics of selected carbon nanotube polymer composites", Carbon, 68, 33-57. doi: http://dx.doi.org/10.1016/j.carbon.2013.11.042

4. Ayache, J., Beaunier, L., Boumendil, J., Ehret, G., Laub, D. "Sample Preparation Handbook for Transmission Electron Microscopy Techniques” Ed. Springer, 2010338 p ISBN 978-1-4419-5975-1

5. Echlin, P. "Handbook of Sample Preparation for Scanning Electron Microscopy and X-Ray Microanalysis" Ed. Springer, 2009330 p ISBN 978-0-387-85731-2

6. Goldstein, J., Newbury, D., Joy, D., Lyman, C., Echlin, P., Lifshin, E., Sawyer, L. \& Michael, J. (2003) Scanning Electron Microscopy and X-Ray Microanalysis, $3^{\text {rd }}$ Ed. Springer

7. Giannuzzi, F.A. "Introduction to Focused Ion Beams" 2005358 p Ed. Springer ISBN 978-0-387-23313-0

8. Osmium plasma coaters http://www.2spi.com/catalog/osmi-coat.html

9. Lillehei, P.T., Kim, J.W., Gibbons, L.J. and Park C., 2009, Nanotechnology, 20, 325708 doi: http://dx.doi.org/10.1088/0957-4484/20/32/325708

10. Zhao, M., Ming, B., Kim, J.W., Gibbons, L.J., Gu, X., Nguyen T., Park, C., Lillehei, P.T., Villarrubia, J.S., Vladár, A.E. and Liddle, A.J., "New Insights into Subsurface Imaging of Carbon Nanotubes in Polymer Composites via Scanning Electron Microscopy” 2015 Nanotechnology doi: http://dx.doi.org/10.1088/0957-4484/26/16/169601

11. SEM-imaging 3D software examples: ImageJ Fiji, Alicona MeX, Digital Surf Mountains, Olympus Scandium, Adobe Photoshop, etc. 